\title{
Hyperosmolality in CHO Culture: Effects on Proteome
}

\author{
Nadiya Romanova ${ }^{1}$, Louise Schelletter ${ }^{1}$, Raimund Hoffrogge ${ }^{1}$, and Thomas Noll ${ }^{1}$ \\ ${ }^{1}$ Bielefeld University Faculty of Technology
}

November 12, 2020

\begin{abstract}
Chinese hamster ovary $(\mathrm{CHO})$ is the most commonly used host cell line for therapeutic protein production. Their exposure to highly concentrated feed solution during fed-batch cultivation can cause an unphysiological osmolality increase $(>300 \mathrm{mOsm} / \mathrm{kg})$ affecting cell physiology, morphology, and proteome. In a companion article "Hyperosmolality in CHO Culture: Effects on Cellular Behavior and Morphology" we show that hyperosmolalities of up to $545 \mathrm{mOsm} / \mathrm{kg}$ force cells to ablate proliferation and gradually increase their volume, almost triplicating it. CHO cells also exhibit a significant hyperosmolality-dependent mitochondrial activity increase. To get a deeper insight into molecular mechanisms involved in these processes, we performed a comparative quantitative label-free proteome study of hyperosmolality-exposed vs. control CHO cells. Our analysis revealed key differentially expressed proteins mediating mitochondrial activation, oxidative stress amelioration, and cell cycle progression. We also discovered a previously unknown strong regulation of proteins altering cell membrane rigidity and permeability. Among others, we detected three members of septins, filamentous proteins forming diffusion barriers in the cell, to be highly upregulated in response to hyperosmolality. Taken together, our observations correlate well with the recent CHO-based fluxome and transcriptome studies and expose new unknown targets involved in response to hyperosmotic pressure in mammalian cells.
\end{abstract}

\section{Hosted file}

Hyperosmolality in CHO culture - Proteome.pdf available at https://authorea.com/users/375355/ articles/492610-hyperosmolality-in-cho-culture-effects-on-proteome

a)

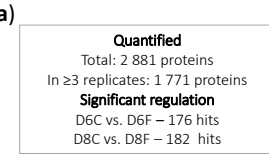

b)

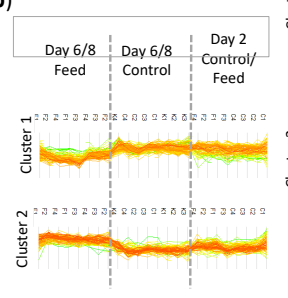

c)

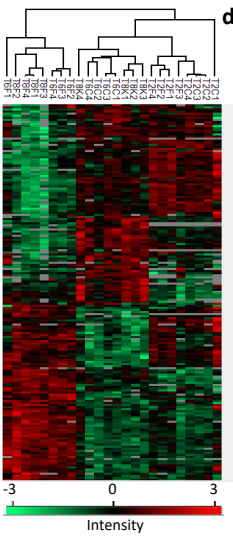

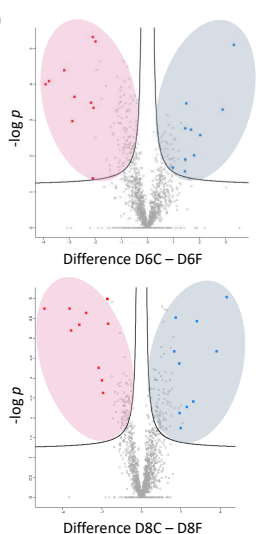




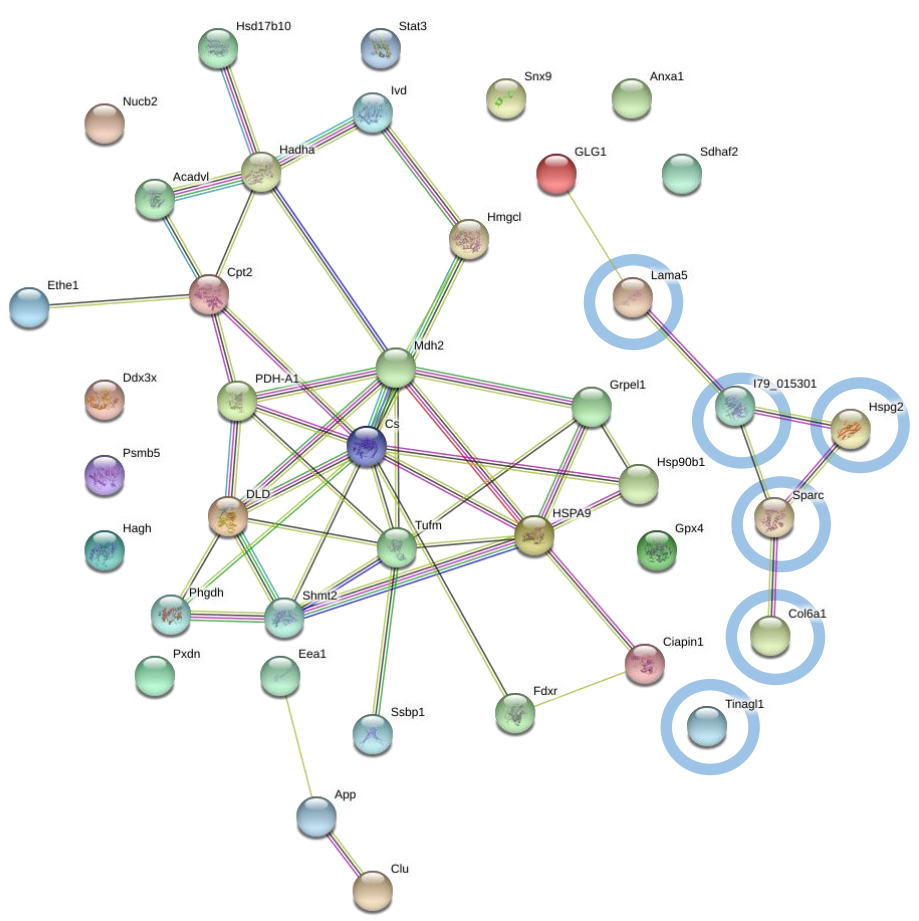

\begin{tabular}{|c|c|c|c|c|}
\hline Node 1 & Node 2 & Node 1 annotation & Node 2 annotation & Confidence score \\
\hline Mdh2 & Cs & Malate dehydrohenase 2 & Cytrate synthase & 0.996 \\
\hline PDH-A1 & DLD & Pyruvate dehydrohenase E1 & Dihydrolipoyl dehydrohenase, $\mathrm{m}$ & 0.991 \\
\hline Acadvl & Hadha & VLC-specific acyl-CoA dehydrogenase & Trifunctional enzyme subunit alpha & 0.967 \\
\hline Grpel1 & HSPA9 & GrpE protein homolog 1 & Stress-70 protein, mitochondrial & 0.964 \\
\hline Acadvl & Cpt2 & VLC-specific acyl-CoA dehydrogenase & Carnitine 0-palmitoyltransferase 2 & 0.961 \\
\hline Ivd & Hadha & Isovaleryl-CoA dehydrogenase & Trifunctional enzyme subunit alpha & 0.954 \\
\hline Hsd17b10 & Hadha & 3-hydroxyacyl-CoA dehydrogenase & Trifunctional enzyme subunit alpha & 0.931 \\
\hline Mdh2 & DLD & Malate dehydrohenase 2 & Dihydrolipoyl dehydrogenase & 0.89 \\
\hline $\mathrm{Hmgcl}$ & Cs & Hydroxymethylglutaryl-CoA lyase & Cytrate synthase & 0.877 \\
\hline |179_015301 & Hspg2 & Nidogen 1.2 & Heparan sulfate core protein & 0.867 \\
\hline Tufm & HSPA9 & Mitochondrial elongation factor Tu & Stress-70 protein, mitochondrial & 0.859 \\
\hline Col6a1 & Sparc & Collagen alpha- $1(\mathrm{VI})$ chain & Kazal-like domain-containing protein & 0.84 \\
\hline Tufm & Mdh2 & Mitochondrial elongation factor Tu & Malate dehydrohenase 2 & 0.828 \\
\hline Shmt2 & DLD & Serine hydroxymethyltransferase & Dihydrolipoyl dehydrohenase, $\mathrm{m}$ & 0.823 \\
\hline Tufm & DLD & Mitochondrial elongation factor $\mathrm{Tu}$ & Dihydrolipoyl dehydrohenase, $\mathrm{m}$ & 0.776 \\
\hline Tufm & Grpel1 & Mitochondrial elongation factor $\mathrm{Tu}$ & GrpE protein homolog 1 & 0.768 \\
\hline 179_015301 & Sparc & Nidogen 1.2 & Kazal-like domain-containing protein & 0.76 \\
\hline App & $\mathrm{Clu}$ & Amyloid-beta A4 protein & Clusterin & 0.736 \\
\hline Hsp90b1 & HSPA9 & HSP90, beta & Stress-70 protein, mitochondrial & 0.734 \\
\hline Mdh2 & Hmgcl & Malate dehydrohenase 2 & Hydroxymethylglutaryl-CoA lyase & 0.711 \\
\hline $\mathrm{Cpt} 2$ & PDH-A1 & Carnitine O-palmitoyltransferase 2 & Pyruvate dehydrogenase $E 1$, subunit $\alpha$ & 0.703 \\
\hline Shmt2 & Tufm & Serine hydroxymethyltransferase & Mitochondrial elongation factor Tu & 0.692 \\
\hline
\end{tabular}

